\title{
From Learning About Machines to Machine Learning: Applications for Mental Health Rehabilitation
}

\author{
Jaya Chaturvedi
}

Published online: 30 March 2020

(C) Springer Nature India Private Limited 2020

Occupational therapy and vocational training for persons with mental health problems traditionally involved working with machines, namely sewing machines for tailoring, printing jobs with industrial printing machines, carpentry with tools, and other vocations involving weaving, making leather products or bakery items, all with the use of small or medium size machines. Machine learning, a relatively newer and advancing concept in health informatics and data analytics, gives an entirely new dimension of potential application for mental health rehabilitation, which is introduced in this guest editorial-Editor-in-Chief's note.

\section{Health Informatics}

Digitalisation of health records has been one of the biggest advances in the field of health informatics. Electronic health records (EHRs) are routinely being utilised to record patient information at point of care. This has led to the creation of a rich data collection source which can be used for a variety of longitudinal studies to understand underlying trends in disease

J. Chaturvedi $(\bowtie)$

Health Informatician and Data Analyst, Department of Biostatistics and Health Informatics, King's College London, London, UK

e-mail: jayachatur@gmail.com progression as well as to inform clinical decision support systems. This can in turn help predict outcomes and alert clinicians of underlying risks based on sound research. EHRs contain combinations of structured and unstructured (free text) fields. The free text within EHRs, especially mental health records, contain a large proportion [1] and variety of information about clinical encounters that might go unnoticed if not explored. This is especially true for mental health records where many presentations, contextual factors, interventions and outcomes are not captured in structured fields (such as symptoms, behaviours, and self-reported experiences) so that the extraction of such information from free text generates a more accurate picture [2]. The potential for data linkages between the hospital EHRs and clinical practices in the community as well as linkages across other population databases [3] provides a more complete picture of the care received by patients.

\section{Chronic Psychiatric Patients and Machine Learning}

Chronic psychiatric patients can hugely benefit from personalisation of treatment and rehabilitation, which can be facilitated by utilising machine learning approaches on the data collected from EHRs to match diverse treatment options to the unique individual profiles of need. This approach will encompass not 
only collecting and integrating large amounts of individual data, but also regularly updating these datasets with new information as it becomes available over the course of treatment [4]. The need for personalisation of treatment is evident in the heterogenicity of people with severe mental illness [5].

Clinical decision support systems can assist in this development of personalised rehabilitation measures by simulating human decision making and combining this with understanding the underlying data of the patients and generating individualised measures based on traits and characteristics of the patients. This will aid clinicians in their judgements and decision making, and provide better, more personalised care to the patients.

\section{Mental Health Data Analytics and Data Mining}

An example of successfully implementing machine learning approaches to mental health data in line with personalisation of treatment is a real-time psychosis risk detection and alerting system based on EHRs [6]. This is currently undergoing feasibility testing for clinical implementation. This system was built using retrospective de-identified patient information from the CRIS database at South London and Maudsley National Health Service Foundation Trust (SLaM) in London, England. The SLaM Biomedical Research Centre Case Register and its Clinical Record Interactive Search (CRIS) application were developed in 2008, generating a research repository of real-time, anonymised, structured and open-text data derived from the electronic health record system used by SLaM, a large mental healthcare provider in southeast London [3]. The psychosis risk detection and alerting system leverages the CogStack platform [7], which is an open-source information retrieval system. CogStack implements data mining techniques such as natural language processing to automate information extraction of medical concepts. CogStack conducts full-text searches of clinical data and carries out realtime calculation of psychosis risk thereby providing early risk-alerting to clinicians and visual monitoring of patients over time.

The role and scope of these developments in health informatics, machine learning, and data analytics for psychosocial rehabilitation of persons with chronic mental health problems has the potential to transform the understanding of the outlook and outcomes in the care of persons with mental illnesses.

\section{References}

1. Stewart R, Soremekun M, Perera G, Broadbent M, Callard F, Denis M, Hotopf M, Thornicroft G, Lovestone S. The South London and Maudsley NHS Foundation Trust Biomedical Research Centre (SLAM BRC) case register: development and descriptive data. BMC Psychiatry. 2009;9:51.

2. Velupillai S, Suominen H, Liakata M, Roberts A, Shah AD, Morley K, Osborn D, Hayes J, Stewart R, Downs J, Chapman W, Dutta R. Using clinical Natural Language Processing for health outcomes research: overview and actionable suggestions for future advances. J Biomed Inform. 2018. https://doi. org/10.1016/j.jbi.2018.10.005.

3. Perera G, Broadbent M, Callard F, Chang C-K, Downs J, Dutta R, Fernandes A, Hayes RD, Henderson M, Jackson R, Jewell A, Kadra G, Little R, Pritchard M, Shetty H, Tulloch A, Stewart R. Cohort profile of the South London and Maudsley NHS Foundation Trust Biomedical Research Centre (SLaM BRC) case register: current status and recent enhancement of an electronic mental health record-derived data resource. BMJ Open. 2016;6(3):e008721.

4. Choi J, Lysaker PH, Bell MD, Dixon L, Margolies P, Gold M, Golden-Roose E, Thime W, Haber LC, Dewberry MJ, Stevens M, Pearlson GD, Fiszdon JM. Decisional informatics for psychosocial rehabilitation: a feasibility pilot on tailored and fluid treatment algorithms for serious mental illness. J Nerv Ment Dis. 2017;205(11):867-72.

5. Spaulding W, Deogun J. A pathway to personalization of integrated treatment: informatics and decision science in psychiatric rehabilitation. Schizophr Bull. 2011;37:S129-37.

6. Wang T, Oliver DAP, Msosa YJ, Fusar-Poli P. Implementation of a real-time psychosis risk detection and alerting system based on electronic health records using CogStack. J Vis Exp. 2020 (in press). https://kclpure.kcl.ac.uk/portal/ en/publications/implementation-of-a-realtime-psychosisrisk-detection-and-alerting-system-based-on-electronichealth-records-using-cogstack(36c30123-f772-4eb5-a4a8c41b19920273)/export.html. Accessed 19 Mar 2020.

7. Jackson R, Kartoglu I, Stringer C, Gorrell G, Roberts A, Song X, Wu H, Agrawal A, Lui K, Groza T, Lewsley D, Northwood D, Folarin A, Stewart R, Dobson R. CogStack-experiences of deploying integrated information retrieval and extraction services in a large National Health Service Foundation Trust hospital. BMC Med Inform Decis Mak. 2018;18(1):47.

Publisher's Note Springer Nature remains neutral with regard to jurisdictional claims in published maps and institutional affiliations. 